Aletheia

ISSN: $1853-3701$

publicaciones@fahce.unlp.edu.ar

Universidad Nacional de La Plata

Argentina

\title{
Políticas trans y acciones afirmativas en los ámbitos universitarios. Conversaciones necesarias para deshacer el cisexismo
}

Radi, Blas

Políticas trans y acciones afirmativas en los ámbitos universitarios. Conversaciones necesarias para deshacer el cisexismo

Aletheia, vol. 10, núm. 19, 2019

Universidad Nacional de La Plata, Argentina

DOI: https://doi.org/10.24215/18521606e026

Esta obra está bajo una Licencia Creative Commons Atribución-NoComercial-Compartirlgual 4.0 Internacional. 
Dossier: Memorias desde las disidencias sexo-genéricas. Preguntas incómodas para desmontar el cisexismo heterosexual.

\title{
Políticas trans y acciones afirmativas en los ámbitos universitarios. Conversaciones necesarias para deshacer el cisexismo
}

\author{
Blas Radi \\ Universidad de Buenos Aires, Argentina \\ blasmradi@gmail.com
}

DOI: https://doi.org/10.24215/18521606e026

Recepción: 01 Noviembre 2019

Aprobación: 20 Noviembre 2019

Recepción: 01 Noviembre 2019

Aprobación: 20 Noviembre 2019

\section{Resumen:}

La elaboración del dossier temático de la revista Aletheia sobre memorias y experiencias de identidades de género disidentes en Argentina se constituyó en una oportunidad para cuestionar y evidenciar problemáticas que recorren y estructuran los debates sobre el tema en el ámbito académico-universitario. Dichas reflexiones se encuentran condensadas en este escrito, un texto dialógico producto de un intercambio reflexivo entre el autor y las coordinadoras del dossier. Blas es profesor de filosofía, becario doctoral (CONICET) y activista de Derechos Humanos. Es docente de Gnoseología de la Facultad de Filosofía y Letras de la Universidad de Buenos Aires y co-coordinador de la Cátedra Libre de Estudios Trans. Este escrito es el producto de conversaciones conjuntas, entrecortadas, pausadas, de preguntas y respuestas construidas a lo largo de los últimos meses. Un diálogo que pretende abrir nuevos interrogantes sobre cómo entendemos, construimos y habitamos los espacios académico-universitarios, qué estrategias se despliegan en la pretendida búsqueda de políticas de inclusión y cuáles son sus limitaciones de las que no podemos escapar. Ello nos conduce a repensar cómo entendemos el género y las identidades.

Palabras clave: Trans, Identidad de género, Universidad.

\section{AbStract:}

The motivation to proposed a new dossier for Aletheia Magazine about memories and experiences of dissident gender identities in Argentina presents great challenges, personal and professional. It drives encourages to dispute questions, to demonstrate the problems that go through span and structure the academic-university environment. These reflections are synthetically found in this paper, that is the result of reflections are synthetically found in this paper, the result of continuous exchanges shared with Blas Radi, in our capacity as coordinators of the dossier. Blas is a professor of philosophy, a doctoral fellow (CONICET) and a Human Rights activist. He is a professor of Gnoseology at the Faculty of Philosophy and Letters of the University of Buenos AiresFacultad de Filosofía y Letras de la Universidad de Buenos Aires and co-coordinator of the Free Chair of Trans Studies. This writing is the product of joint exchanges, often paused and disperse; of questions and answers built together over the last moths. This writing is the product of joint conversations, choppy, leisurely, of questions and answers built over the last months. A dialogue that seeks to open new questions about how we understand, build and inhabit academic-university spaces, what strategies are deployed in the intended search for inclusion policies and what are their limitations that we cannot escape. This leads us to rethink how we understand gender and identities.

KEYWORDS: Trans, Gender identity, University.

\section{¿Cuáles son los problemas que encuentran las personas trans en el ámbito ACADÉMICO?}

Esta pregunta merece algunas aclaraciones, tal vez más que de las que voy a hacer. Agradezco que la hayan formulado porque nos da la oportunidad de desplegar su complejidad. Para hacer un inventario de los problemas que encuentran las personas trans en cualquier ámbito, como mínimo tenemos que saber de quiénes estamos hablando. Y aunque parezca un contrasentido esto no es tan sencillo. 
"Trans" es un término polisémico que suele funcionar en simultáneo de maneras distintas: en su uso coloquial, funciona de manera operativa (cuando explicitamos que lo vamos a usar como una campana semántica para hacer referencia a todas las personas que se identifican con un género distinto al asignado) y también funciona como identidad de género (cuando lo aplicamos para aludir a aquellas personas que se identifican a sí mismas de ese modo). Esto significa que tenemos un término y dos conceptos en uso, que además aplican a universos distintos, lo que sienta las bases para un problema de ambigüedad: no todas las personas trans en el sentido operativo, se identifican a sí mismas como "trans".

Entonces, ¿̇a quiénes nos referimos cuando hablamos de "las personas trans"? Con demasiada frecuencia las políticas públicas, las investigaciones, las noticias y hasta la memoria colectiva se deslizan de un concepto a otro sin explicitarlo. Este problema tiene consecuencias. Se expresa, por ejemplo, cuando da forma a las políticas y expectativas institucionales que asumen que "las personas trans" son un grupo identificable y relativamente homogéneo, por lo menos con relación a ciertas características.

Claro que esto no es así. Y la diversidad de experiencias incide en los modos de relacionarse con la academia. Reforcemos esto: no todas las personas trans son visibles como tales, no todas cambian sus documentos, no todas tienen una expresión de género que conforma las expectativas sociales depositadas sobre su identidad, no todas transicionan a la misma edad ni en el mismo momento de la carrera universitaria o formación profesional, no todas son estudiantes.

Hago esta aclaración porque muchas veces la relación entre "las personas trans" y las instituciones se resuelve con la fórmula de la expulsión, que hace imposible que las personas trans estudien. Este es un problema muy grave pero no es el único.

Para pensar en términos muy generales podemos tomar a la transfobia y el cisexismo como claves para dar sentido las experiencias de todas las personas que se identifican con un género distinto al asignado en las instituciones en general y en la academia en particular. Abarcamos así distintos problemas: aquellos que son perpetrados por individuos particulares, que son explícitos y deliberados, y también los que son estructurales, que tal vez no respondan a la voluntad de una persona con malas intenciones.

Estos problemas se presentan en las relaciones interpersonales, en los trámites administrativos, en el currículum, en los requisitos de acceso a becas y a oportunidades de desarrollo académico, en la constitución de los institutos, en el desarrollo de las investigaciones, incluso en la forma de entender lo que es el género y la violencia de género. En fin, el catálogo de problemas es larguísimo.

\section{¿A QUÉ TE REFERís CON LOS PROBlemas DERIVADOS DE LA MANERA DE ENTENDER EL GÉNERO?}

"Género" es un término talismán, es decir, una palabra cargada de cierto prestigio -que impregna los discursos cada vez que hace aparición-y hace muy difícil que éstos sean puestos en tela de juicio. Pero, ¿de qué estamos hablando cuando hablamos de género? ¿Decimos siempre lo mismo? ¿Estamos todxs de acuerdo? Las mismas preguntas valen para la "perspectiva de género". Hoy es una referencia ineludible en ciertos ámbitos de la academia progresista. Lo que no parece tan ineludible es la tematización de su significación epistemológica y sus implicancias ético políticas.

En los últimos años experimentamos grandes transformaciones en nuestras costumbres, hábitos, e instituciones con relación al género. Y no sólo al género, también en el modo de entender el sexo y la sexualidad. El impacto y las ramificaciones de estos cambios se han extendido por todo el tejido social, erosionando el significado corriente de dichos conceptos en favor de recursos interpretativos que, por un lado, capturan de manera más apropiada la diversidad de las experiencias humanas y, por el otro, se adecuan al derecho internacional de derechos humanos. Nuestra Ley de Identidad de Género (L.N n 26.743), por ejemplo, adopta el concepto de "identidad de género" provisto por los Principios de Yogyakarta (un 
documento del año 2007 que aplica los derechos humanos específicamente a cuestiones de orientación sexual e identidad de género).

De acuerdo con los Principios, la identidad de género es una vivencia interna, una experiencia subjetiva que es independiente de las características sexuales, del sexo asignado al nacer y de la información consignada en el documento de identidad. Aunque la definición es clara todavía no se ha desvanecido la presencia de sentidos correspondientes a una forma de vida anterior. Por el contrario, una cierta inercia los mantiene vivos, generando tensiones y desafíos dentro de las instituciones, que también alcanzan a la universidad. La institución y muchxs agentes institucionales suponen y refuerzan el sentido común del género: los sexos son dos, los géneros también y hay una relación de necesidad biológica entre ambos.

En general se trata de personas que saben bien poco de biología pero apelan a ella como si se tratara de un manual de instrucciones del mundo que recupera una a una sus opiniones. Temas tales como la arquitectura de los baños y los contenidos curriculares de la educación sexual han sido y son también una fuente inagotable de polémicas. No son las únicas pero funcionan como indicadores del cisexismo estructural de las instituciones y permiten hacer explícitos los compromisos respecto del género de lxs agentes que forman parte de ellas.

Por ejemplo, en la teoría feminista la afirmación del género como construcción social convive armónicamente con la afirmación de la bioanatomía como fundamento y como verdad. Como señala Mauro Cabral en un texto genial: en su adopción feminista, género sólo se predica de mujeres y varones (cis) y de la relación de desigualdad entre ambos. En consecuencia, la perspectiva de género sólo ve mujeres y varones cis. Esto es un gran problema. Uno doblemente grave dado que con demasiada frecuencia se asume que la perspectiva de género es la solución universal a todos los problemas (Ver Cabral, 2011).

\section{Y, EN CONCRETO, ¿QUÉ TIPO DE PROBLEMAS GENERA?}

Voy a responder con un ejemplo. Este año en la Facultad de Filosofía y Letras de la UBA se creó una agrupación feminista que presentó un proyecto de paridad de género en el Departamento de la carrera de Filosofía y también en el Consejo directivo de la Facultad. El proyecto fue cambiando de forma. Primero establecía un "cupo femenino inclusivo". Era inclusivo porque incorporaba a lesbianas y personas trans. La idea inicial era que el $50 \%$ de la composición de las comisiones evaluadoras o comités organizadores de eventos esté conformado por un $50 \%$ de identidades femeninas y/o de lesbianas y trans como mínimo.

Fui invitado a adherir y compartí el proyecto con el equipo de la Cátedra Libre de Estudios Trans. Nos pareció positivo que se promuevan medidas de acción afirmativa con una perspectiva sensible a la existencia de personas trans. De hecho el proyecto hacía referencia a la Ley de Identidad de Género.

Sin embargo, encontramos también algunos problemas que presentamos como preocupaciones y críticas constructivas. Tiene sentido pensar en ellas para revisar qué concepto de género está en juego y qué consecuencias tienen nuestros compromisos teóricos sobre este tipo de disposiciones institucionales.

Una de las preocupaciones que señalamos apuntaba a la composición de ese "cupo inclusivo”. De acuerdo con el proyecto el cupo comprendía a un conjunto de personas compuesto por aquellas "(auto)percibidas como mujeres, lesbianas, trans y travestis". El problema que tienen este tipo de estrategias es que al no considerar a las personas trans como grupo que pueda ser desagregado, aunque puedan ser satisfactorias para poner un límite al sexismo institucional, son insuficientes para desmontar el cisexismo -que gobierna todos los espacios, incluso aquellos que están conformados exclusivamente por mujeres cis. De hecho el anexo del proyecto incluía ejemplos en los que este cupo se había producido espontáneamente aún sin ser establecido. Se señalaba ahí que en unas Jornadas de Filosofía, la Comisión organizadora había alcanzado "la paridad de género". No obstante, el 100\% de lxs integrantes de la Comisión son personas cis. Señalamos entonces que si todas las comisiones y comités tuvieran la misma composición identitaria que la Comisión Organizadora de las Jornadas de Filosofía eso tendría un impacto positivo en la paridad entre varones y mujeres cis (y estaría 
libre del primer problema que señalamos), pero para las personas trans no haría la diferencia. Y no sólo eso, se generaría una ficción de paridad sobre una base profundamente desigual, lo cual sería altamente perjudicial.

Por eso, desde la Cátedra decidimos no acompañar la propuesta pero nos ofrecimos a colaborar para pensar en una alternativa capaz de superar estas limitaciones. Lamentablemente no tuvimos oportunidad de hacerlo y el proyecto se aprobó de todos modos, aunque se introdujeron modificaciones. Por ejemplo, en lugar de establecer un cupo se decidió implementar un "techo" que limite la participación de varones cis a no más del 50\%. Esto sigue siendo un problema, especialmente para los varones trans porque deberían explicitar que son trans (recordemos la distinción que hicimos entre los dos significados del término). De no hacerlo, serían registrados como cis y aplicaría sobre ellos el recorte del techo.

Para que esto quede, claro volvamos al comienzo de la entrevista: Despejada la ambigüedad semántica respecto del término trans (en la primera pregunta), queda claro que no todas las personas trans se identifican como "trans". Dicho con otras palabras: no todas las personas trans hacen público que son personas trans. Y esto tiene mucho sentido porque con frecuencia revelar esta información puede tener y de hecho tiene consecuencias muy negativas sobre sus condiciones de existencia, incluso en la Facultad. Quienes tenemos la experiencia de haber hecho la carrera siendo abiertamente trans podemos dar cuenta de ello. Y no debemos remontarnos al pasado remoto para encontrar ejemplos. Ahora mismo en la Facultad de Filosofía y Letras hay una docente del Instituto de Género que se burla de estudiantes trans por redes sociales.

No es por nada que nuestra Ley de Identidad de Género (L.N. 26.743) hace foco en la confidencialidad. Si la confidencialidad es un valor fundamental en las políticas trans lo es, justamente, por este tipo de problemáticas que experimentamos las personas trans cuando hacemos público que somos trans. La política del techo, entonces, pondría a los varones trans en situación de tener que hacer público su estatuto transicional o privarse de acceder al beneficio. El primer caso implica la renuncia a la confidencialidad y, por tanto, la exposición a los efectos negativos que esto trae aparejado. El segundo los deja por fuera de la política afirmativa.

Entonces, acá tenemos un ejemplo muy claro de cómo se traducen en la práctica los problemas derivados de formas diferentes de entender el género. Y tenemos también un desafío: diseñar políticas afirmativas que no impliquen una violación a la Ley de Identidad de Género.

\section{¿Qué postura ADOPTA EL MOVIMiento TRANS ReSPECTO DE ESTOS PROBLEMAS CON LA ACADEMIA?}

No hay una postura coherente. Por momentos parece que la educación superior es simultáneamente un derecho que debe ser garantizado y un mal que es preferible evitar. En este escenario hay quienes reclaman tanto el acceso a la educación como el reconocimiento de las personas trans como productoras de conocimiento. En general describen a las personas trans como personas que en virtud de una gran cantidad de obstáculos han tendido a quedar por fuera de las instituciones educativas y demandan acceso a ellas. Esta descripción se ajusta a la experiencia de muchísimas personas trans.

Pero al mismo tiempo, hay quienes critican a las personas trans que acceden a la educación universitaria, justamente por el hecho de haber estudiado. También hay personas trans estudiando, dando clases, o haciendo investigación. Aquí muchas veces hay un desplazamiento que hace de toda persona trans académica una persona "academicista". A menudo las personas que sostienen estas posturas contradictorias son las mismas (y a veces son personas trans). No pasa lo mismo con las personas cis, pero en el caso de las personas trans, si estudian o han estudiado, suelen ser descalificadas como personas privilegiadas, que como tales no merecen atención y hasta merecen repudio.

Esto nos devuelve de alguna manera a la primera pregunta, bajo la forma de una de las paradojas que desarrolla Wendy Brown (2002): la descripción que se da de las personas trans como personas que sistemáticamente ven violado su derecho a la educación está formulada de una manera que no les 
permite escapar de esa violación. Por otra parte, pretender que las personas trans sean reconocidas como agentes epistémicos mientras se las desconoce deliberadamente como tales constituye una contradicción performativa.

Desde la Cátedra Libre lo que hacemos es habilitar un espacio de reflexión, investigación y transferencia dentro del campo de los Estudios Trans. Conformamos un equipo de trabajo, tenemos encuentros periódicos de lectura y discusión, ofrecemos cursos abiertos y gratuitos, y colaboramos con procesos institucionales dentro de la Facultad (como el del cupo). Igualmente, a pesar de dejar en claro que se trata de una iniciativa académica, justamente porque es tan difícil entender que las personas trans pueden motorizar y ser parte de un espacio semejante, todavía hay quienes asumen que se trata de una agrupación política o un espacio terapéutico que ofrece contención.

\section{REFERENCIAS}

AA.VV. (2007). Principios de Yogyakarta sobre la aplicación de la legislación internacional de derechos humanos en relación con la orientación sexual y la identidad de género. Disponible en: http://yogyakartaprinciples.org/princ iples-sp/about/

Brown, W. (2002). Suffering rights as paradoxes. Constellations. Número 7. p. 208-229.

Cabral, M. (2011). “La paradoja transgénero” En Carlos Cáceres, María Esther Mogollón, Griselda Pérez-Luna \& Fernando Olivos (eds.), Sexualidad, ciudadania y derechos humanos en América Latina: un quinquenio de aportes regionales al debate y la reflexión (pp. 97-104). Lima: Instituto de Estudios en Salud, Sexualidad y Desarrollo Humano \& Universidad Peruana Cayetano Heredia.

Ley Nacional de Identidad de Género n 26.743. (2012). Disponible en: https://www.argentina.gob.ar/sites/default /files/ley-26.743-identidad-de-genero_0.pdf

\section{BY-NC-SA}

